Ann. Zootech., I972, 21 (3), 479-483.

NOTE TECHNIQUE

\title{
RECHERCHE D'UNE MESURE PRÉCISE DE L'ÉPAISSEUR DE LA PEAU CHEZ LE POULET
}

\author{
F.-H. RICARD \\ Station expérimentale d'Aviculture du Magneraud, I. N. R. A., \\ 17 - Surgères \\ RÉSUMÉ
}

Trois micromètres ayant des caractéristiques différentes ont été comparés en vue d'obtenir une mesure précise de la peau du ponlet, mesure effectuée au niveau de l'aile sur la carcasse plumée. Le meilleur résultat (répétabilité d'une mesure égale à 0,83 ) a été obtenu avec un appareil muni d'un ressort souple et de disques larges. Cette technique a permis, en particulier, de mettre en évidence une variabilité génétique entre familles dans une souche de poulets de chair.

\section{INTRODUCTION}

La finesse de la peau, chez les volailles, est souvent considérée comme une caractéristique de qualité qu'il faudrait améliorer. Mais il s'agit d'un caractère relativement complexe qui n'a jamais fait l'objet, du moins à notre connaissance, d'une étude approfondie. A priori, on peut penser que l'épaisseur constitue un élément simple important de la finesse de la peau. C'est cette épaisseur que nous avons cherché à estimer chez le poulet. Dans un premier travail nous a vons étudié plusieurs techniques de mesure (RICARD, I968). Les résultats obtenus n'étaient pas entièrement satisfaisants, la répétabilité de la mesure étant en général peu élevée. Cependant une mesure faite au niveau de l'aile paraissait liée au poids total de peau de la carcasse et pouvait donc être utilisée comme une estimation indirecte de ce dernier. A la suite de cette observation, nous avons cherché à améliorer la technique de mesure en essayant des appareils exerçant une pression plus faible sur la peau et munis d'un cadran plus précis. Nous donnons ici les résultats obtenus. 


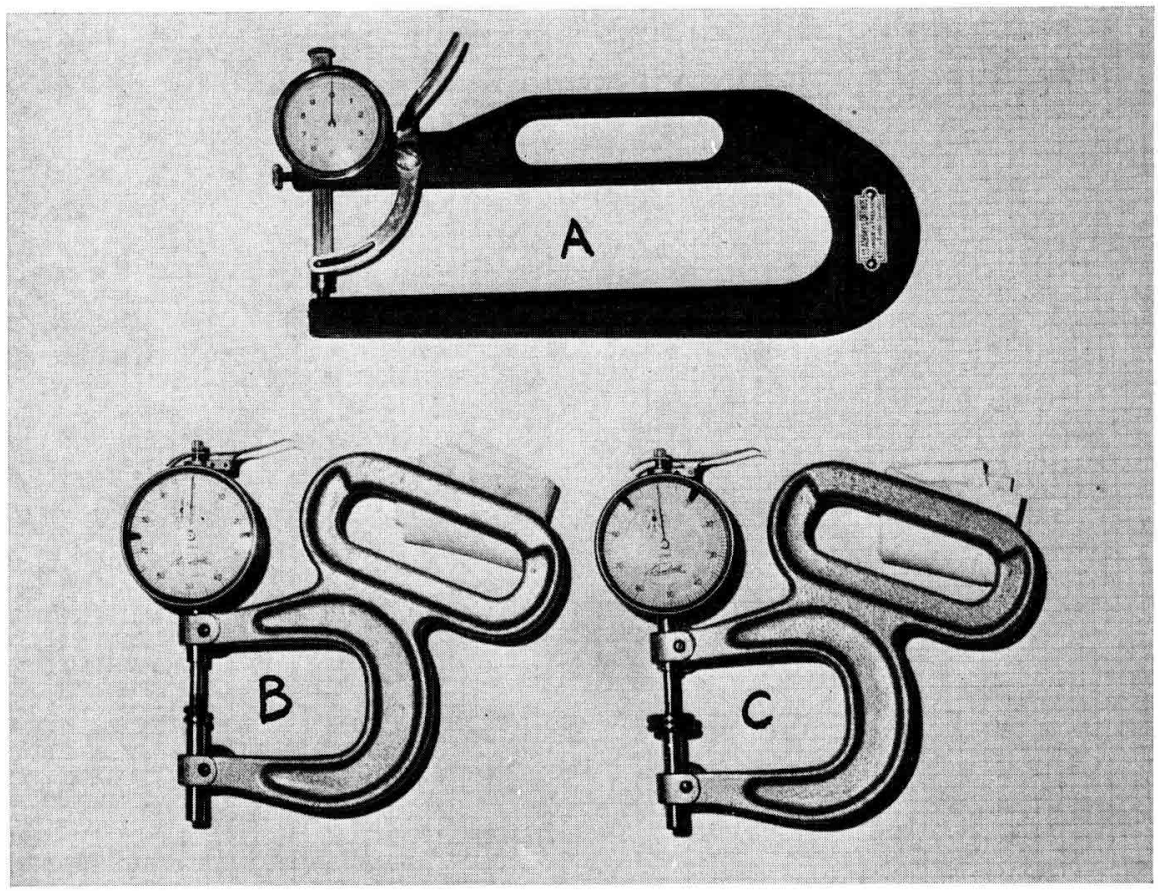

FIG. I. - Vue des trois micromètres étudiês

(Les carrés du support ont $5 \mathrm{~mm}$ de côté)

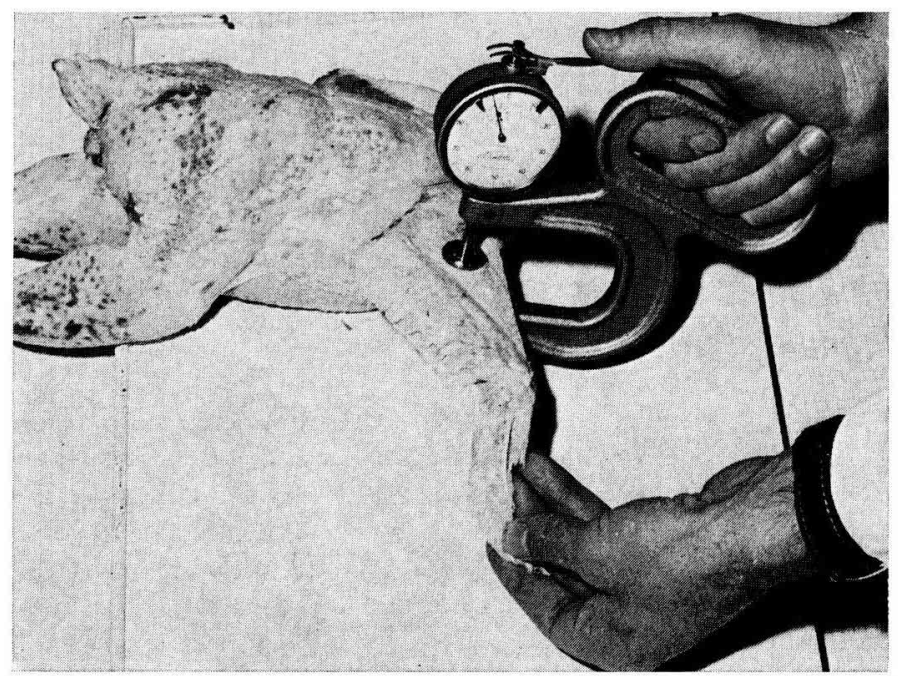

Fig. 2. - Mise en ceuvre de l'appareil $C$

pour la mesure de l'épaisseur de la peau au niveau de l'aile 


\section{MATÉRIEL E'T MÉTHODES}

Nous avons comparé les 3 appareils qui sont représentés sur la figure $I$ et dont les caractéristiques sont indiquées dans le tableau I. L’appareil A est celui utilisé lors de notre essai précédent (RICARD, I968). L'appareil B est un micromètre de construction un peu différente, plus maniable, muni d'un ressort beaucoup plus souple et dont le cadran permet une lecture au centième de millimètre. L'appareil C est identique à $\mathrm{B}$ mais il est muni de disques plus larges (I9 mm au lieu de 8).

TABLEAU I

Caractéristiques des appareils étudiés

\begin{tabular}{|c|c|c|c|}
\hline & Appareil A & Appareil B & Appareil C \\
\hline Poids de l'appareil $(g)$ & 801 & 471 & 481 \\
\hline Diamètre utile des disques $(\mathrm{mm})$. & 10 & 8 & 19 \\
\hline $\begin{array}{l}\text { Force relative du ressort (évalua- } \\
\text { tion approximative) } \ldots \ldots \ldots \ldots\end{array}$ & 6 & 1 & 1 \\
\hline Graduation du cadran ........ & $1 / 20 \mathrm{~mm}$ & $1 / 100 \mathrm{~mm}$ & $1 / 100 \mathrm{~mm}$ \\
\hline
\end{tabular}

Nous nous sommes limité ici à l'étude de l'épaisseur de la membrane alaire, c'est-à-dire à la double épaisseur de peau comprise entre les deux premiers segments de l'aile. La façon de procéder est illustrée par la figure 2. Les mesures ont été faites sur un lot de $4^{\circ}$ poulets âgés de 66 jours : 20 coquelets d'un poids vif moyen de $2203 \mathrm{~g}$ et 20 poulettes d'un poids vif moyen de I $724 \mathrm{~g}$. Sur l'aile droite de chaque poulet, on a effectué 3 répétitions de la mesure avec chacun des 3 appareils. Une analyse de variance des résultats a été faite séparément pour chaque appareil et la répétabilité pour une mesure, $\rho_{1}$, a été estimée par le coefficient de corrélation intra-classe. Le modèle utilisé était le suivant:

\begin{tabular}{l|c|c|c}
\hline \hline Source de variation & $\begin{array}{c}\text { Degrés de } \\
\text { liberté }\end{array}$ & $\begin{array}{c}\text { Composition } \\
\text { du carré moyen }\end{array}$ & Répétabilité \\
\hline & & & \\
Totale $\ldots \ldots \ldots \ldots \ldots$ & 119 & $\sigma_{e}^{2}+3 \sigma_{a}^{2}$ & $\rho_{1}=\frac{\sigma_{a}^{2}}{\sigma_{a}^{2}+\sigma_{e}^{2}}$ \\
Poulet $\ldots \ldots \ldots \ldots \ldots$ & 89 & $\sigma_{e}^{2}$ & \\
Résiduelle $\ldots \ldots \ldots \ldots$ & 80 & \\
\hline
\end{tabular}

\section{RÉSUL'TATS ET DISCUSSION}

Les résultats obtenus sont indiqués dans le tableau 2. Les différences observées pour les moyennes peuvent s'expliquer par les caractéristiques des appareils. L'appareil A, muni d'un ressort puissant, comprime plus la peau, d'où une épaisseur plus faible. Avec l'appareil B, qui est muni de disques étroits, on recouvre plus ou moins bien les follicules plumaires présents sur la membrane alaire. Il donne des valeurs plus faibles que l'appareil C. Ce dernier permet de recouvrir une large surface de peau, comprenant toujours plusieurs follicules plumaires. Malgré ces différences de moyennes, l'examen des coefficients de variation montre que les 3 appareils conduisent à une variabilité phénotypique équivalente. 
La répétabilité obtenue avec l'appareil A est meilleure pour cet essai que pour le précédent (RICARD, I968) où nous avions trouvé une valeur de o,54 seulement. La répétabilité relativement faible observée avec l'appareil $B$ semble due à un diamètre insuffisant des disques. En effet, l'appareil $\mathrm{C}$, qui est du même type mais possède des disques plus larges, permet d'obtenir une répétabilité bien meilleure (cf. tabl. 2). On peut considérer qu'une mesure est précise quand sa répétabilité atteint une valeur de l'ordre de 0,9 . Dans le cas présent, pour obtenir ce chiffre, il faut effectuer 3 répétitions avec l'appareil A, 6 avec l'appareil B et 2 avec l'appareil C.

\section{TABLEAU 2}

Comparaison des résultats obtenus aveo chaque appareil

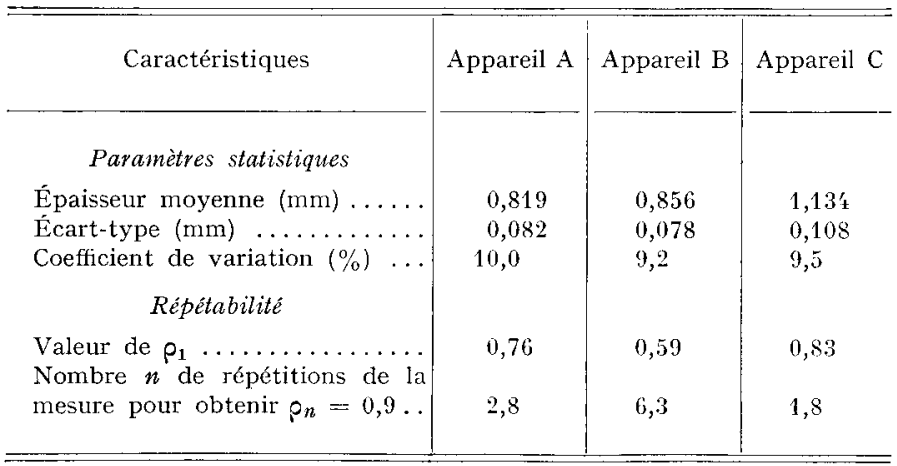

En plus des résultats qui viennent d'être analysés, il faut tenir compte du fait que les appareils $B$ et $C$ sont d'un maniement plus commode que l'appareil A. Nous pouvons donc conclure que la mesure de l'épaisseur de la peau à l'aide de l'appareil C est une technique très satisfaisante pour des travaux de laboratoire, surtout s'il est possible de répéter deux fois la mesure.

A titre d'application, nous donnons les résultats d'un calcul d'héritabilité effectué dans une souche de poulets de chair à croissance moyenne. L'épaisseur de la peau de l'aile a été mesurée à l'aide de l'appareil C ( 2 répétitions) sur un ensemble de I 358 coquclets issus de 74 pères et 305 mères, obtenus en 4 générations successives et abattus à l'âge de 80 jours. L'analyse hiérarchique des variances a permis de mettre en évidence des différences hautement significatives entre familles. La composante mère de l'héritabilité était égale à o,6o et la composante père égale à 0,37 .

Reçu pour publication en mars 1972.

\section{SUMMARY}

\section{SEARCHING FOR AN ACCURATE MEASUREMENT OF SKIN THICKNESS IN THE CHICKEN}

Three micrometer-type apparatus have been compared for appraising skin thickness in chickens. Measurements were made at the wing level. The best apparatus gave a repeatibility value of 0.83 for one measurement. Its main characteristics were : smooth spring, wide disks and. 
a dial giving hundredths of millimeters (see table $\mathbf{I}$ and figure $\mathrm{I}$, apparatus $\mathrm{c}$ ). By using the above method, genetic variability of skin thickness appeared between families of a strain of broiler chickens.

\section{RÉFÉRENCE BIBIOGRAPHIQUE}

Ricard F. H., 1968. Essais d'estimation de l'épaisseur et de l'importance de la peau chez le poulet. Ann. Zootech., 17, 459-466. 